\title{
NECESSARY AND SUFFICIENT CONDITIONS FOR THE OSCILLATIONS OF A MULTIPLICATIVE DELAY LOGISTIC EQUATION
}

BY

S. R. GRACE (Dept. of Engineering Mathematics, Cairo University, Orman, Giza, 12000, Egypt)

I. GYÖRI (Dept. of Math. and Computing, Univ. of Veszprim, P.O. Box 158, Hungary)

AND

B. S. LALLI (Dept. of Math. and Statistics, University of Saskatchewan, Saskatoon, S7N 0W0, Canada)

Abstract. Necessary and sufficient conditions are presented for the oscillation of all positive solutions of the multiplicative delay logistic equation

$$
\frac{d N(t)}{d t}=r(t) N(t)\left(1-\prod_{j=1}^{m}\left(\frac{N\left(g_{j}(t)\right.}{K}\right)\right)
$$

about the positive equilibrium $K$. The cases when $r(t)=r$ and $g_{j}(t)=t-\tau_{j}$ or $g_{j}(t)=\left[t-k_{j}\right],[\cdot]$ denoting the greatest integer function, where $r, \tau_{j}$, and $k_{j}$ are positive constants, $j=1,2, \ldots, m$, are also included.

1. Introduction. A wide class of population growth models is described by the logistic equation

$$
\frac{d y(t)}{d t}=r y(t)\left(1-\frac{y(t)}{K}\right),
$$

where $r$ and $K$ are positive parameters; $r$ is the intrinsic growth rate whose density is denoted by $y(t)$ in (1.1) and $K$ denotes the carrying capacity. Elementary analysis of (1.1) indicates that solutions of (1.1) are monotonic functions of $t$ with $\lim _{t \rightarrow \infty} y(t)=K$ if $y(0)>0$. It has been observed that it is difficult to fit observed data to a logistic curve and one of the problems noted by Smith [13] is due to the fact that (1.1) has not adequately taken into account the time delays in the growth rate response to changes in the densities of the population. Temporal oscillations have been observed in the densities of populations even in controlled environments and such oscillations have been ascribed to time delays in growth rate responses (Nicholson [11, 12], Caperon [1], Maynard Smith [10]). Since solutions of (1.1) do not show

Received August 24, 1992.

1991 Mathematics Subject Classification. Primary 34CXX.

This work was done during the summer of 1992 when the authors were visiting the University of Saskatchewan as visiting Professors of Mathematics.

(C)1995 Brown University 
some of the experimentally observed oscillations of laboratory populations, (1.1) has been modified to the delay logistic equation, namely

$$
\frac{d y(t)}{d t}=r y(t)\left(1-\frac{y(t-\tau)}{K}\right)
$$

by Hutchinson [6]. The time delay $\tau$ (assumed to be a positive parameter) in (1.2) can cause oscillations in solutions of (1.2) about the positive equilibrium $K$ and there have been numerous investigations of the qualitative behavior of (1.2) and several of its generalizations; for example, see Jones [7], Kakutani and Markus [8], and Wright [15].

In an attempt to introduce several environmental negative feedback effects, (1.2) has been modified and generalized to the form

$$
\frac{d y(t)}{d t}=r y(t)\left(1-\frac{\sum_{j=1}^{m} a_{j} y\left(t-\tau_{j}\right)}{K}\right)
$$

in which $a_{j}$ and $\tau_{j}$ are nonnegative numbers, $j=1,2, \ldots, m$. For a recent contribution to the study of (1.3), we refer to the papers of Gopalsamy [2], Györi and Ladas [4], and Lenhart and Travis [9]. It is not known whether several environmental intraspecific negative feedback effects act additively as in (1.3) or multiplicatively as in

$$
\frac{d y(t)}{d t}=r y(t)\left(1-\prod_{j=1}^{m}\left(\frac{y\left(t-\tau_{j}\right)}{K}\right)\right) .
$$

In [3], Gopalsamy and Lalli derived sufficient conditions for all positive solutions of (1.4) to oscillate about $K$ and for a global attractivity of the positive equilibrium of (1.4). The authors have been motivated to consider the multiplicative delay logistic equation

$$
\frac{d N(t)}{d t}=r(t) N(t)\left(1-\prod_{j=1}^{m}\left(\frac{N\left(g_{j}(t)\right)}{K}\right)\right),
$$

which is of some mathematical interest as a generalization of (1.2) and (1.4). The functions $r(t)$ and $g_{j}(t)$ are assumed to be locally bounded and locally integrable for $t \geq t_{0} \geq 0$, and $j=1,2, \ldots, m$. Because of the weaker conditions on the argument $g_{j}(t), j=1,2, \ldots, m$, equations of type (1.5) with piecewise continuous arguments, namely

$$
\frac{d N(t)}{d t}=r N(t)\left(1-\prod_{j=1}^{m}\left(\frac{N\left(\left[t-k_{j}\right]\right)}{K}\right)\right)
$$

where $k_{j}$ and $r$ are positive constants, $j=1,2, \ldots, m$, are considered as a special case of Eq. (1.5) and consequently, the oscillatory behavior of all positive solutions of Eq. (1.6) about $K$ follows from the corresponding results for Eq. (1.5).

For recent contributions to the study of the oscillatory behavior of differential equations with piecewise continuous arguments, we refer to the paper of Cooke and Weiner [14] and Györi and Ladas [4], and the references cited therein. 
We also mention that the delay $t-g_{j}(t), j=1,2, \ldots, m$ is not assumed to be bounded and hence our results include the unbounded time delay too. Of particular interest, the results to be presented are applicable to equations of the form

$$
\frac{d N(t)}{d t}=r(t) N(t)\left(1-\prod_{j=1}^{m}\left(\frac{N\left(\gamma_{j} t\right)}{K}\right)\right),
$$

where $\gamma_{j}$ is a constant and $0<\gamma_{j} \leq 1, j=1,2, \ldots, m$.

The purpose of this paper is to establish necessary and sufficient conditions for the oscillation of all positive solutions of Eqs. (1.4)-(1.6) about $K$ via comparison with first-order delay equations of the type

$$
\dot{z}(t)+r(t) \sum_{j=1}^{m} z\left(g_{j}(t)\right)=0
$$

where the functions $r$ and $g_{j}, j=1,2, \ldots, m$ are assumed as in the equations considered.

2. Some lemmas. In this section, we establish some lemmas that will be used in the proof of the main results.

Consider the delay differential inequality

$$
\dot{x}(t)+\sum_{j=1}^{m} p_{j}(t) x\left(g_{j}(t)\right) \leq 0
$$

and the equation

$$
\dot{y}(t)+\sum_{j=1}^{m} p_{j}(t) y\left(g_{j}(t)\right)=0,
$$

where $g_{j}, p_{j}:\left[t_{0}, \infty\right) \rightarrow(0, \infty)$ are locally bounded and locally integrable functions, $j=1,2, \ldots, m, g_{j}(t) \leq t$ for $t \geq t_{0} \geq 0$, and $\lim _{t \rightarrow \infty} g_{j}(t)=\infty$, $j=1,2, \ldots, m$. Next, we let

$$
T^{*}=\min _{1 \leq j \leq m}\left\{\inf _{t \geq t_{0}} g_{j}(t)\right\} .
$$

By a solution on $\left[t_{0}, \infty\right)$ of inequality (2.1) we mean a continuous function $x(t)$ defined on $\left[T^{*}, \infty\right)$, which is absolutely continuous and which satisfies inequality (2.1) a.e. on $\left[t_{0}, \infty\right)$. As usual, a solution of inequality (2.1) or Eq. (2.2) is said to be oscillatory if it has arbitrarily large zeros and nonoscillatory otherwise.

LEMma 2.1 . If the inequality $(2.1)$ has a positive solution on $\left[T^{*}, \infty\right)$, then equation (2.2) has a positive solution on $\left[T^{*}, \infty\right)$.

Proof. Let $x(t)$ be a nonoscillatory solution of inequality (2.1), say $x(t)>0$ for $t \geq T^{*}$. Define

$$
\alpha(t)=\frac{-\dot{x}(t)}{x(t)} \quad \text { for } t \geq t_{0}
$$

Then

$$
x(t)=x\left(t_{0}\right) \exp \left(-\int_{t_{0}}^{t} \alpha(s) d s\right)
$$


and from (2.1), we get

$$
\alpha(t) \geq \sum_{j=1}^{m} \frac{x\left(g_{j}(t)\right)}{x\left(T_{j}(t)\right)} p_{j}(t) \exp \left(\int_{T_{j}(t)}^{t} \alpha(s) d s\right), \quad t \geq t_{0},
$$

where

$$
T_{j}(t)=\max \left\{t_{0}, g_{j}(t)\right\}
$$

Define a sequence $\beta_{k}(t)(k=0,1, \ldots)$ as follows:

$$
\begin{aligned}
& \beta_{0}(t)=0 \quad \text { for } t \geq t_{0} \quad \text { and } \\
& \beta_{k+1}(t)=\sum_{j=1}^{m} \frac{x\left(g_{j}(t)\right)}{x\left(T_{j}(t)\right)} p_{j}(t) \exp \left(\int_{T_{j}(t)}^{t} \beta_{k}(s) d s\right) \quad \text { for } t \geq t_{0},
\end{aligned}
$$

$k=0,1,2, \ldots$.

It is easy to see from (2.3) and (2.4) that

$$
0 \leq \beta_{0}(t) \leq \beta_{1}(t) \leq \cdots \leq \beta_{k}(t) \leq \beta_{k+1}(t) \leq \alpha(t), \quad t \geq t_{0} .
$$

The pointwise limit of the sequence $\left\{\beta_{k}(t)\right\}$ as $k \rightarrow \infty$ exists and if we let

$$
\alpha(t) \geq \beta(t)=\lim _{t \rightarrow \infty} \beta_{k}(t), \quad t \geq t_{0},
$$

then by the Lebesgue Dominated Convergence Theorem, we conclude that the function $\beta(t)$ is locally integrable and

$$
\int_{T_{j}(t)}^{t} \beta_{k}(s) d s \rightarrow \int_{T_{j}(t)}^{t} \beta(s) d s \quad \text { as } k \rightarrow \infty .
$$

Now, it is easy to check that the positive function defined by

$$
y(t)= \begin{cases}x\left(t_{0}\right) \exp \left(-\int_{t_{0}}^{t} \beta(s) d s\right), & t \geq t_{0}, \\ x(t), & T^{*} \leq t \leq t_{0},\end{cases}
$$

satisfies Eq. (2.2) on $\left[t_{0}, \infty\right)$. This completes the proof.

LEMMA 2.2. If the solutions of Eq. (2.2) are oscillatory, then

$$
\int_{t_{0}}^{\infty} p_{j}(s) d s=\infty \quad \text { for some } j=1,2, \ldots, m
$$

Proof. Suppose that Eq. (2.2) is oscillatory. If

$$
\int_{t_{0}}^{\infty} p_{j}(s) d s<\infty \quad \text { for all } j=1,2, \ldots, m,
$$

then there exists a $T \geq t_{0}$ such that

$$
q=\int_{T}^{\infty} \sum_{j=1}^{m} p_{j}(s) d s<1
$$

Let

$$
M=\frac{1}{1-q}(>1) .
$$


Now, define a sequence $\left\{y_{k}(t)\right\}$ as follows:

$$
\begin{gathered}
y_{0}(t)=1 \quad \text { for } T^{*} \leq t<\infty, \\
y_{k+1}(t)= \begin{cases}1+\int_{t}^{\infty} \sum_{j=1}^{m} p_{j}(s) y_{k}\left(g_{j}(s)\right) d s & \text { for } t \geq T, \\
1 & \text { for } T^{*} \leq t<T,\end{cases}
\end{gathered}
$$

$k=0,1,2, \ldots$.

It can easily be seen that

$$
1 \leq y_{0}(t) \leq y_{1}(t) \leq \cdots \leq y_{k}(t) \leq y_{k+1}(t) \leq M,
$$

and hence $y(t)=\lim _{t \rightarrow \infty} y_{k}(t)$ exists and

$$
y(t)= \begin{cases}1+\int_{t}^{\infty} \sum_{j=1}^{m} p_{j}(s) y\left(g_{j}(s)\right) d s, & \text { for } t \geq T, \\ 1 & \text { for } T^{*} \leq t<T,\end{cases}
$$

and clearly $y(t)$ is positive and satisfies Eq. (2.2), a contradiction. This completes the proof.

For the autonomous case, i.e., when $p_{j}(t)=q_{j}$ and $g_{j}(t)=t-\tau_{j}$, where $q_{j}$ and $\tau_{j}$ are positive constants, $j=1,2, \ldots, m$, the following lemma is needed, which is proved in [4, Theorem 3.2.2].

LEMMA 2.3. The following statements are equivalent.

(a) The delay differential equation (2.2) has a positive solution.

(b) There exists $\delta_{0} \in(0,1)$ such that for every $\delta \in\left[0, \delta_{0}\right]$ the delay equation

$$
\dot{z}(t)+(1-\delta) \sum_{j=1}^{m} q_{j} z\left(t-\tau_{j}\right)=0
$$

has a positive solution.

Finally, we prove the following lemma for the case when $p_{j}(t)=q_{j}$ and $g_{j}(t)=$ $\left[t-k_{j}\right]$, where $q_{j}$ and $k_{j}$ are positive constants, $j=1,2, \ldots, m$, and [.] denotes the greatest integer function.

LEMMA 2.4. The following statements are equivalent.

(a) Eq. (2.2) has a positive solution.

(b) There exists a $\delta_{0} \in(0,1)$ such that for every $\delta \in\left[0, \delta_{0}\right]$, the equation

$$
\dot{z}(t)+(1-\delta) \sum_{j=1}^{m} q_{j} z\left(\left[t-k_{j}\right]\right)=0
$$

has a positive solution.

Applying the results of Györi and Ladas [5], it suffices to prove that the following statements are equivalent.

(A) The characteristic equation

$$
\lambda-1+\sum_{j=1}^{m} q_{j} \lambda^{-k_{j}}=0
$$

has no real root. 
(B) There exists a $\delta_{0} \in(0,1)$ such that for all $\delta \in\left[0, \delta_{0}\right]$ the characteristic equation

$$
\lambda-1+(1-\delta) \sum_{j=1}^{m} q_{j} \lambda^{-k_{j}}=0
$$

has no real root.

Proof. Clearly $(B) \Rightarrow(A)$. To prove $(A) \Rightarrow(B)$, set

$$
F(\lambda)=\lambda^{k+1}-\lambda^{k}+\sum_{j=1}^{m} q_{j} \lambda^{k-k_{j}}=0,
$$

where $k=\max _{1 \leq j \leq m} k_{j}$, and assume that $(2.8)$ has no real root in [0,1]. Therefore, the $\min _{0 \leq \lambda \leq 1} F(\lambda)$ exists and is a positive number $c$ (say). Hence,

$$
F(\lambda) \geq c \quad \text { for all } \lambda \in[0,1] .
$$

Assume that for some $\delta>0$ Eq. (2.7) has a real root, say $\mu_{\delta}$. It follows from (2.7) that

$$
\left(F\left(\mu_{\delta}\right)=\right) \mu_{\delta}^{k+1}-\mu_{\delta}^{k}+\sum_{j=1}^{m} q_{j} \mu_{\delta}^{k-k_{j}}=\delta \sum_{j=1}^{m} q_{j} \mu_{\delta}^{k-k_{j}} .
$$

Now, it is easy to check that

$$
\max _{0 \leq \mu \leq 1} \delta \sum_{j=1}^{m} q_{j} \mu^{k-k_{j}}=\delta \sum_{j=1}^{m} q_{j}=\delta Q>0 .
$$

Choose $\delta$ so that $0<\delta<\frac{c}{2 Q}$. Thus, the minimum of the left-hand side of (2.9) is $c$ and the maximum of the right-hand side of (2.9) is less than $\frac{c}{2}$, a contradiction. This completes the proof.

3. Main results. Consider the multiplicative delay logistic equation

$$
\frac{d N(t)}{d t}=r(t) N(t)\left(1-\prod_{j=1}^{m}\left(\frac{N\left(g_{j}(t)\right)}{K}\right)\right),
$$

where $r, g_{j}:\left[t_{0}, \infty\right) \rightarrow(0, \infty)$ are locally bounded and locally integrable functions, $t_{0} \geq 0, g_{j} \leq t$, and $\lim _{t \rightarrow \infty} g_{j}(t)=\infty, j=1,2, \ldots, n$, and $K$ is a positive constant.

We assume that Eq. (1.5) is provided with initial conditions of the form

$$
N(s)=\phi(s) \geq 0, \quad \phi(0)>0, \quad \phi \in C\left(\left[T^{*}\left(t_{0}\right), t_{0}\right],[0, \infty)\right)
$$

where

$$
T^{*}\left(t_{0}\right)=\min _{1 \leq j \leq m}\left\{\inf _{t \geq t_{0}} g_{j}(t)\right\} .
$$

One can verify that all solutions of Eq. (1.5) are defined for all $t \geq t_{0} \geq 0$ and remain positive for $t \geq t_{0}$. We note that a positive solution of Eq. (1.5) is said to be oscillatory about $K$ if there exists a sequence $\left\{t_{n}\right\}, t_{n} \rightarrow \infty$ as $n \rightarrow \infty$ such that

$$
N\left(t_{n}\right)-K=0, \quad n=1,2, \ldots,
$$


and $N$ is said to be nonoscillatory about $K$ if there exists $T_{0} \geq 0$ such that

$$
|N(t)-K|>0 \quad \text { for } t \geq T_{0} .
$$

Now, since $N(t)>0$, we can define $z$ as follows:

$$
z(t)=\ln \left(\frac{N(t)}{K}\right)
$$

and note that the oscillation and nonoscillation of $N$ about $K$ is equivalent to that of $z$ about zero. Equations (1.5) and (3.1) together lead to

$$
\frac{d z(t)}{d t}=r(t)\left(1-\exp \left(\sum_{j=1}^{m} z\left(g_{j}(t)\right)\right)\right) .
$$

The following result provides sufficient conditions for the oscillation of all positive solutions of Eq. (1.5) about $K$.

THEOREM 3.1. Every positive solution of Eq. (1.5) oscillates about $K$ if, for all sufficiently small $\delta \geq 0$, every solution of the equation

$$
\dot{y}(t)=-r(t)(1-\delta) \sum_{j=1}^{m} y\left(g_{j}(t)\right)
$$

oscillates about zero.

Proof. Assume that Eq. (3.2) has a nonoscillatory solution $z$. Then either

$$
z(t)>0 \quad \text { or } \quad z(t)<0 \quad \text { for } t \geq T \text { for some } T \geq t_{0} \geq 0 .
$$

In both cases, one can easily see that the functions $z(t)$ and $\dot{z}(t)$ are bounded on $[T, \infty)$, and as a consequence of the nonoscillatory character of $z(t)$ in Eq. (1.5)

$$
\lim _{t \rightarrow \infty} z(t)=\gamma \text { exists . }
$$

We claim that $\gamma=0$. To prove it, assume that $\gamma \neq 0$. Now, if $z(t)>0$ for $t \geq T$, then $z(t)$ decreases to $\gamma>0$, and hence there exists a $T_{1} \geq T$ so that

$$
1-\exp \left(\sum_{j=1}^{m} z\left(g_{j}(t)\right)\right) \leq 1-e^{\gamma m}=-c, \quad c>0 \quad \text { and } \quad t \geq T_{1} .
$$

Integrating Eq. (3.2) from $T_{1}$ to $t$ we have

$$
z(t)=z\left(T_{1}\right)+\int_{T_{1}}^{t} r(s)\left(1-\exp \left(\sum_{j=1}^{m} z\left(g_{j}(s)\right)\right)\right) d s .
$$

Thus,

$$
z(t) \leq z\left(T_{1}\right)-c \int_{T_{1}}^{t} r(s) d s .
$$

By applying Lemma 2.2 to Eq. (3.3), we see that $\int_{T_{1}}^{t} r(s) d s \rightarrow \infty$ as $t \rightarrow \infty$ and hence

$$
z(t) \rightarrow-\infty \quad \text { as } t \rightarrow \infty,
$$


a contradiction. The proof of the case $z(t)<0$ for $t \geq T$ is similar and we omit the details. This proves the claim.

Next, suppose that $z(t)>0$ for $t \geq T$. Then Eq. (3.2) leads to

$$
\begin{aligned}
\dot{z}(t) & =r(t)\left(\frac{1-\exp \left(\sum_{j=1}^{m} z\left(g_{j}(t)\right)\right)}{\sum_{j=1}^{m} z\left(g_{j}(t)\right)}\right) \sum_{j=1}^{m} z\left(g_{j}(t)\right) \\
& \leq-r(t) \sum_{j=1}^{m} z\left(g_{j}(t)\right), \quad t \geq T .
\end{aligned}
$$

Applying Lemma 2.1, we see that the equation

$$
\dot{y}(t)+r(t) \sum_{j=1}^{m} y\left(g_{j}(t)\right)=0
$$

has a positive solution, a contradiction.

Finally, we suppose that $z(t)<0$ for $t \geq T$. We let

$$
-z(t)=v(t)>0 \quad \text { for } t \geq T .
$$

Then

$$
\dot{v}(t)=r(t)\left(\frac{\exp \left(-\sum_{j=1}^{m} v\left(g_{j}(t)\right)-1\right)}{\sum_{j=1}^{m} v\left(g_{j}(t)\right)}\right) \sum_{j=1}^{m} v\left(g_{j}(t)\right) .
$$

Since $v(t) \rightarrow 0$ monotonically as $t \rightarrow \infty$, there exists $\delta \in(0,1)$ and $T_{1} \geq T$ so that

$$
\dot{v}(t) \leq-r(t)(1-\delta) \sum_{j=1}^{m} v\left(g_{j}(t)\right), \quad t \geq T_{1} .
$$

Once again, we apply Lemma 2.1 and obtain the desired contradiction. This completes the proof.

The following theorem provides necessary conditions for the oscillation of all solutions of Eq. (1.5) about $K$.

Theorem 3.2. If the delay equation (1.8) is nonoscillatory, then there exists a $T \geq t_{0}$ such that Eq. (1.5) has a solution that is nonoscillatory about $K$.

Proof. Let $z(t)$ be a nonoscillatory solution of Eq. (1.8), say $z(t)>0$ for $t \geq t_{0}$. There exists a $t_{1} \geq t_{0}$ so that $z\left(g_{j}(t)\right)>0$ for $t \geq t_{1}$. Choose $T \geq t_{1}$ so that

$$
T^{*}(T)=\min _{1 \leq j \leq m}\left\{\inf _{t \geq T} g_{j}(t)\right\} \geq t_{0} .
$$

Clearly,

$$
\dot{z}(t) \leq 0 \text { exists a.e. on }\left[T^{*}(T), \infty\right)
$$

Now, define

$$
\lambda_{1}(t)=-\frac{\dot{z}(t)}{z(t)}>0 \quad \text { for } t \geq T^{*}(T)
$$


Integrating (3.4) from $T^{*}(T)$ to $t$, we obtain

$$
z(t)=z\left(T^{*}(T)\right) \exp \left(\int_{T^{*}(T)}^{t}-\lambda(s) d s\right) .
$$

Using (3.5) in equations (1.8) and (3.4), we have

$$
\lambda_{1}(t)=r(t) \sum_{j=1}^{m} \exp \left(\int_{g_{j}(t)}^{t} \lambda_{1}(s) d s\right) .
$$

Now, we define the function sequence $\left\{\alpha_{k}\right\}$ and $\left\{y_{k}\right\}$ as follows:

$$
\begin{gathered}
\alpha_{0}(t)= \begin{cases}0 & \text { for } t \geq T, \\
\lambda_{1}(t) & \text { for } T^{*}(T) \leq t \leq T,\end{cases} \\
\alpha_{k+1}(t)= \begin{cases}r(t)\left(\frac{1-\exp \left(-\sum_{j=1}^{m} y_{k}\left(g_{j}(t)\right)\right)}{\sum_{j=1}^{m} y_{k}\left(g_{j}(t)\right)}\right) \sum_{j=1}^{m} \exp \left(\int_{g_{j}(t)}^{t} \alpha_{k}(s) d s\right) & \text { for } t \geq T \\
\lambda_{1}(t) & \text { for } T^{*}(T) \leq t \leq T,\end{cases}
\end{gathered}
$$

and

$$
y_{k}(t)=z\left(T^{*}(T)\right) \exp \left(-\int_{T^{*}(T)}^{t} \alpha_{k}(s) d s\right), \quad k=0,1,2, \ldots
$$

Clearly

$$
0 \leq \alpha_{0}(t) \leq \lambda_{1}(t)
$$

From the definition of $\alpha_{0}(t)$ and $\alpha_{1}(t)$ it follows that

$$
\begin{aligned}
\lambda_{1}(t) & =r(t) \sum_{j=1}^{m} \exp \left(\int_{g_{j}(t)}^{t} \lambda_{1}(u) d u\right) \\
& \geq r(t) \sum_{j=1}^{m} \exp \left(\int_{g_{j}(t)}^{t} \alpha_{0}(u) d u\right) \\
& \geq r(t)\left(\frac{1-\exp \left(-\sum_{j=1}^{m} y_{0}\left(g_{j}(t)\right)\right)}{\sum_{j=1}^{m} y_{0}\left(g_{j}(t)\right)}\right) \sum_{j=1}^{m} \exp \left(\int_{g_{j}(t)}^{t} \alpha_{0}(s) d s\right) \\
& =\alpha_{1}(t) \geq \alpha_{0}(t) \geq 0
\end{aligned}
$$

and

$$
\begin{aligned}
0 \leq y_{1}(t) & =\exp \left(-\int_{T^{*}(T)}^{t} \alpha_{1}(s) d s\right) \\
& \leq \exp \left(-\int_{T^{*}(T)}^{T} \alpha_{0}(s) d s\right) \\
& =y_{0}(t) .
\end{aligned}
$$

Inductively, one can easily obtain

$$
0 \leq \alpha_{0}(t) \leq \alpha_{1}(t) \leq \cdots \leq \alpha_{k}(t) \leq \alpha_{k+1}(t) \leq \lambda_{1}(t)
$$


and

$$
y_{0}(t) \geq y_{1}(t) \geq \cdots \geq y_{k}(t) \geq 0 \quad \text { for } t \geq T^{*}(T) .
$$

Proceeding as in the proof of Lemma 2.1 , one can easily check that the limit functions of the sequences $\left\{\alpha_{k}(t)\right\}$ and $\left\{y_{k}(t)\right\}$, say $\alpha(t)$ and $y(t)$, respectively, satisfy the equation

$$
\dot{y}(t)+r(t)\left(1-\exp \left(-\sum_{j=1}^{m} y\left(g_{j}(t)\right)\right)=0,\right.
$$

and hence Eq. (1.5) has a positive nonoscillatory solution about $K$. This completes the proof.

Next, we are concerned with necessary and sufficient conditions for the oscillation of all positive solutions of Eqs. (1.4) and (1.6) about $K$.

Corollary 3.1. Consider Eq. (1.4) and let $\tau=\max _{1 \leq j \leq m} \tau_{j}$. The following statements are equivalent:

(a) Every solution $N:[-\tau, \infty) \rightarrow(0, \infty)$ of Eq. (1.4) oscillates about $K$.

(b) Every solution of the delay equation

$$
\dot{z}(t)+r \sum_{j=1}^{m} z\left(t-\tau_{j}\right)=0
$$

oscillates about zero.

Proof. It follows from the proof of Theorems 1 and 2, Lemma 2.3, and the fact

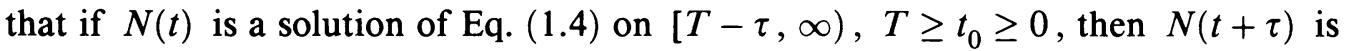
also a solution of Eq. (1.4) on $[-\tau, \infty)$.

COROllary 3.2. Consider Eq. (1.6) and let $k=\max _{1 \leq j \leq m} k_{j}$. The following statements are equivalent.

(c) Every solution $N:[-k, \infty) \rightarrow(0, \infty)$ of Eq. (1.6) oscillates about $K$.

(c) Every solution of the equation

$$
\dot{z}(t)+r \sum_{j=1}^{m} z\left(\left[t-k_{j}\right]\right)=0
$$

oscillates about zero.

Proof. It follows from the proof of Theorems 1 and 2, Lemma 2.4, and the fact that if $N(t)$ is a solution of Eq. (1.6) on $\left[K^{*}-k, \infty\right), K^{*}$ is a large integer, then $N\left(t+K^{*}\right)$ is also a solution of Eq. (1.6) on $[-k, \infty)$.

REMARKS. Using some known results for the oscillation of Eq. (2.6) (e.g., [4, Theorem 2.2.1]), one can easily check that Theorem 2.2 in [3] is included in Corollary 3.1 .

4. Some general comments. It is worthwhile to mention that there are a number of oscillation results related to linear delay equations with continuous and with piecewise continuous arguments (see, e.g., [4, 5]). Hence, by applying our linearized oscillation results one can easily establish sufficient and necessary conditions for the positive solutions of Eq. (1.5) to be oscillatory about the steady state $K$. Especially if $r(t)$ 
and $g_{j}(t), j=1,2, \ldots, m$, are continuous functions and the delays $\sigma_{j}(t)=t-g_{j}(t)$ satisfy

$$
\liminf _{t \rightarrow \infty} \int_{\sigma(t)}^{t} r(u) d u \geq \frac{1}{e}, \quad \sigma(t)=\max _{1 \leq j \leq m}\left\{\sigma_{j}(t)\right\},
$$

then for all sufficiently small $\delta>0$, every solution of Eq. (3.3) oscillates about zero (see, e.g., [2]) and hence every positive solution of Eq. (1.5) oscillates about $K$.

If $r(t)=p([t])$ and $g_{j}(t)=\left[t-k_{j}[t]\right]$, where [.] denotes the greatest integer function, $p: N \rightarrow[0, \infty)$ and $k_{j}: N \rightarrow N, 1 \leq j \leq m(N=\{0,1, \ldots\})$ are given sequences, then condition (4.1) can be replaced by

$$
\liminf _{n \rightarrow+\infty} \sum_{i=n-k}^{n-1} p(i)>\left(\frac{k}{k+1}\right)^{k+1},
$$

where $k=\max k_{j}$. This will also ensure the oscillation of the positive solutions of Eq. (1.5) about $K$.

It is clear that if the intrinsic growth rate $r(t)$ is constant and the delay terms $\sigma_{j}(t)$ and $k_{j}(n)$ are large enough then (4.1) and (4.2), respectively, are satisfied and hence the positive solutions of (1.5) oscillate about $K$. One can interpret this in such a way that the long memory causes oscillation in the population growth.

Acknowledgments. The authors express their sincere thanks to the referee for very helpful comments and suggestions.

\section{REFERENCES}

[1] J. Caperon, Time lag in population growth response of Isochrysis Galbana to a variable nitrate environment, Ecology 5, 188-192 (1969)

[2] K. Gopalsamy, Global stability in the delay logistic equation with discrete delays, Houston J. Math (to appear)

[3] K. Gopalsamy and B. S. Lalli, Oscillatory and asymptotic behavior of a multiplicative delay logistic equation, Dynamics and Stability of Systems 7, 35-42 (1992)

[4] I. Györi and G. Ladas, Oscillation theory of Delay Differential Equations, Oxford Science Publications, Oxford, 1991

[5] I. Györi and G. Ladas, Linearized oscillations for equations with piecewise constant arguments, Differential and Integral Equations 2, 123-131 (1989)

[6] G. E. Hutchinson, Circular causal systems in ecology, Ann. New York Acad. Sci. 50, 221-246 (1948)

[7] G. S. Jones, On the nonlinear differential difference equation $f^{\prime}(x)=f(x-1)[1+f(x)]$, J. Math. Anal. Appl. 4, 440-469 (1962)

[8] S. Kakutani and L. Markus, On the nonlinear difference differential equation $\dot{y}(t)=$

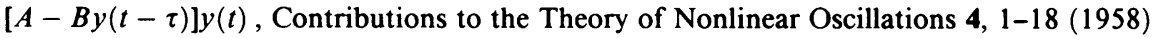

[9] S. M. Lenhart and C. C. Travis, Global stability of a biological model with time delay, Proc. Amer. Math. Soc. 96, 75-78 (1986)

[10] J. Maynard Smith, Models in Ecology, Cambridge Univ. Press, Cambridge, 1974

[11] A. J. Nicholson, An outline of the dynamics of animal populations, Austral. Zool. 2, 9-65 (1954)

[12] A. J. Nicholson, The self adjustment of populations to change, Cold Spring Harbor, Symposium on Qualitative Biology 22, 153-173 (1957)

[13] E. E. Smith, Quantitative aspects of population growth in the dynamic of growth processes (E. J. Boel, ed.), Princeton Univ. Press, Princeton, N.J., 1954

[14] K. L. Cooke and J. Weiner, A survey of differential equations with piecewise continuous arguments, Lecture Notes in Mathematics, vol. 1475 (S. Busenberg and M. Martelli, eds.), Springer-Verlag, Berlin-Heidelberg-New York, 1991

[15] E. M. Wright, A nonlinear difference differential equation, J. Reine Angew. Math. 194, 66-87 (1955) 